PROCEEDINGS OF THE

AMERICAN MATHEMATICAL SOCIETY

Volume 125, Number 11, November 1997, Pages 3391-3396

S 0002-9939(97)04187-7

\title{
THE GEODESIC FLOW GENERATES A FAST DYNAMO: AN ELEMENTARY PROOF
}

\author{
C. CHICONE AND Y. LATUSHKIN
}

(Communicated by Jeffrey B. Rauch)

\begin{abstract}
We give elementary and explicit arguments to show that the geodesic flow on the unit tangent bundle of a two dimensional Riemannian manifold with constant negative curvature provides an example of a "fast" dynamo for the magnetic kinematic dynamo equation.
\end{abstract}

\section{INTRODUCTION}

Consider a steady state solution $\mathbf{v}$ of Euler's equation for an ideal fluid with pressure $p$ on a three dimensional space $X$ :

$$
\frac{d \mathbf{v}}{d t}+(\mathbf{v}, \nabla) \mathbf{v}=-\operatorname{grad} p, \quad \operatorname{div} \mathbf{v}=0 .
$$

The kinematic dynamo equation for the induction of a magnetic field $\mathbf{u}$ by the fluid velocity field $\mathbf{v}$ is given by

$$
\dot{\mathbf{u}}=\varepsilon \triangle \mathbf{u}+\operatorname{curl}(\mathbf{v} \times \mathbf{u}), \quad \operatorname{div} \mathbf{u}=0,
$$

where $\varepsilon=R_{m}^{-1}$, and $R_{m}$ is the magnetic Reynolds number.

Let $\mathcal{D}_{\varepsilon}$ denote the linear operator defined by $(2)$ in $L_{2}(X, \mu)$ for a volume $\mu$ on $X$ invariant with respect to the flow $\varphi^{t}$ induced by $\mathbf{v}$ :

$$
\mathcal{D}_{\varepsilon} \mathbf{u}:=\varepsilon \Delta \mathbf{u}+\operatorname{curl}(\mathbf{v} \times \mathbf{u}) .
$$

Also, note that $\mathcal{D}_{\varepsilon}$ depends on the choice of the volume and the metric on $X$. Define the spectral bound for $\mathcal{D}_{\varepsilon}$ by $s_{\varepsilon}:=\sup \left\{\operatorname{Re} \lambda: \lambda \in \sigma\left(\mathcal{D}_{\varepsilon}\right)\right\}$. If $s_{\varepsilon}>0$ for all sufficiently small positive $\varepsilon$, then $\mathbf{v}$ is called a kinematic dynamo. The dynamo is called "fast" if, in addition, $\lim \sup _{\varepsilon \rightarrow 0} s_{\varepsilon}>0$.

There exist several examples of fast dynamos; see $[3,5,11,14,16]$ and the literature cited therein. In particular, by a deep result of M. M. Vishik [15], every Anosov flow with smooth foliations on a compact three dimensional manifold produces a fast dynamo. The proof of this result uses the full range of hyperbolic theory together with the theory of asymptotic expansions for pseudo differential operators. These general methods do not seem to provide explicit formulas for the eigenvalues of $\mathcal{D}_{\varepsilon}$.

Received by the editors April 24, 1996.

1991 Mathematics Subject Classification. Primary 76W05, 58F99, 58G25.

Key words and phrases. Kinematic dynamo, geodesic flow.

The first author's research was supported by the National Science Foundation under the grant DMS-9303767; the second author was supported by the National Science Foundation under the grant DMS-9400518 and by the SRF of the University of Missouri. 
In the present note we give an elementary and explicit example of an Anosov flow that is a steady state solution of Euler's equation and that produces fast dynamo action. In fact, let $M$ denote a smooth two dimensional compact Riemannian manifold without boundary that has constant negative Gauss curvature $k$ and let $X$ denote the total space of its unit tangent bundle. The set $X$ is a compact three dimensional manifold. For the appropriate natural choice of Riemannian metric (Sasaki metric) and volume element on $X$, the geodesic vector field $\mathbf{v}$, the vector field on $X$ that generates the geodesic flow, is a steady state solution of Euler's equation with $\operatorname{grad} p=0$. This vector field produces the fast dynamo. In fact, we will prove the following theorem:

Theorem 1.1. If $\mathbf{v}$ generates the geodesic flow on the total space of the unit tangent bundle $X$ of a two dimensional compact Riemannian manifold $M$ with constant negative curvature $k$ and if $\mathcal{D}_{\varepsilon}$ denotes the associated dynamo operator defined with respect to the Sasaki metric and volume on $X$, then, for each magnetic Reynolds number $R_{m}>\sqrt{-k}$, the dynamo operator $\mathcal{D}_{\varepsilon}$ has the positive eigenvalue

$$
\lambda_{\varepsilon}:=\frac{1}{2}\left[-\varepsilon\left(1+k^{2}\right)+\sqrt{\varepsilon^{2}\left(1-k^{2}\right)^{2}-4 k}\right],
$$

and $\limsup _{\varepsilon \rightarrow 0} s_{\varepsilon} \geq \lim _{\varepsilon \rightarrow 0} \lambda_{\varepsilon}=\sqrt{-k}>0$. In particular, $\mathbf{v}$ is a steady state solution of Euler's equation that induces a fast kinematic dynamo.

It is, of course, well known that the geodesic flow on the unit tangent bundle of a two-dimensional Riemanian manifold with constant negative curvature provides an example of a fast dynamo. More general results appear in the papers by M. Vishik, [15] and [16, Section 9]. Also, we suspect that the authors of [3] were aware of this fact. However, the purpose of this paper is to provide a simple elementary proof of this result and, especially, to obtain the explicit formula (4) for the positive eigenvalue of $\mathcal{D}_{\varepsilon}$. Our proof of Theorem 1.1 uses only elementary formulas from vector calculus and certain commutation relations for Lie brackets obtained by L. Green in [12]; see also the related papers $[6,7,8,9,13]$.

\section{Proof of THE THEOREM}

We recall the construction of the parallelization of $X$. Let $g$ be the Riemannian metric on $M$ and let $T_{m} M$ denote the tangent plane at $m \in M$ with the scalar product $g_{m}(\cdot, \cdot)$ induced by $g$. The total space of the unit tangent bundle of $M$ is the set $X$,

$$
X:=\left\{(m, \mathbf{w}): m \in M, \mathbf{w} \in T_{m} M, g_{m}(\mathbf{w}, \mathbf{w})=1\right\} .
$$

This set has the natural structure of a three dimensional compact manifold.

For $\gamma: \mathbb{R} \rightarrow M$, a curve in $M$, we let $\dot{\gamma}(t) \in \mathcal{T}_{\gamma(t)} M$ denote the tangent vector to $\gamma$ at $\gamma(t)$. If $x=(m, \mathbf{w}) \in X$, then there is a unique geodesic $\gamma: \mathbb{R} \rightarrow M$, such that $\gamma(0)=m$ and $\dot{\gamma}(0)=\mathbf{w}$. The geodesic flow $\varphi^{t}$ in $X$ is defined as follows: If $x=(m, \mathbf{w}) \in X$ and $\gamma$ denotes the geodesic starting at $m$ with tangent vector $\mathbf{w}$, then $\varphi^{t} x=(\gamma(t), \dot{\gamma}(t))$.

The geodesic vector field $\mathbf{v}$ is defined to be the generator of the flow $\varphi^{t}$. We note that $\mathbf{v}$ has no zeros on $X$. There are two additional nonvanishing vector fields on $X$, that we denote by $\mathbf{y}$ and $\mathbf{z}$, such that for each $x \in X$ the set $\{\mathbf{v}(x), \mathbf{y}(x), \mathbf{z}(x)\}$ is a basis for the tangent space $T_{x} X$. These vector fields are defined as follows. Since $M$ is orientable, there is a well-defined notion of positive rotation through a right angle. 
That is, there is a well-defined map $(m, \mathbf{w}) \mapsto\left(m, \mathbf{w}^{\perp}\right)$ such that $g_{m}\left(m, \mathbf{w}^{\perp}\right)=0$ and $g_{m}\left(\mathbf{w}^{\perp}, \mathbf{w}^{\perp}\right)=g_{m}(\mathbf{w}, \mathbf{w})$. We define $\mathbf{y}$ as the generator of the flow $(m, \mathbf{w}) \mapsto$ $\varphi^{t}\left(m, \mathbf{w}^{\perp}\right)$ on $X$. Also, let $r_{m}(t) \mathbf{w}=e^{i t} \mathbf{w}$ give the unit speed rotation of the unit circle in the positive sense relative to the orientation in $T_{m} M$. Define $\mathbf{z}$ as the generator of the fiber rotation flow on $X$ given by $(m, \mathbf{w}) \mapsto\left(m, r_{m}(t) \mathbf{w}\right)$.

We use the notation $[\mathbf{u}, \mathbf{v}]=\mathbf{u v}-\mathbf{v u}$ for the Lie bracket of the vector fields $\mathbf{u}, \mathbf{v}$ on $X$ and we let $k$ denote the lift of the Gauss curvature of $M$ to $X$. The following bracket relations, as reported in [12], are valid:

$$
[\mathbf{v}, \mathbf{y}]=k \mathbf{z}, \quad[\mathbf{v}, \mathbf{z}]=-\mathbf{y}, \quad[\mathbf{y}, \mathbf{z}]=\mathbf{v} .
$$

There is a unique Riemannian metric on $X$ defined by declaring, for each $x \in X$, the set $\{\mathbf{v}(x), \mathbf{y}(x), \mathbf{z}(x)\}$ to be an orthonormal basis for $T_{x} X$. We will denote the induced scalar product in $T_{x} X$ by $\langle\cdot, \cdot\rangle$. It can be shown that this Riemannian metric coincides with the Sasaki metric (see [8] for a definition), but this fact will not be needed here.

Let $\mu$ denote the volume element on $X$ chosen so that $\mu(\mathbf{v}, \mathbf{y}, \mathbf{z})=1$ and let $\omega_{\mathbf{u}}(\boldsymbol{\xi})=\langle\boldsymbol{\xi}, \mathbf{u}\rangle$. Using the standard (see, e.g., [1, 2]) notations $\mathrm{d}$ and $\mathrm{i}$ for the exterior and the interior derivative, we recall that the maps $\mathbf{u} \mapsto \omega_{\mathbf{u}}$ and $\mathbf{u} \mapsto \mathrm{i}_{\mathbf{u}} \mu$ are isomorphisms and that the operations of vector analysis on $X$ correspond to the exterior differential calculus applied to the target differential forms. For example, the operators curl and div are defined by

$$
\mathrm{d} \omega_{\mathbf{u}}=\mathrm{i}_{\text {curl } \mathbf{u}} \mu, \quad \mathrm{di}_{\mathbf{u}} \mu=\operatorname{div} \mathbf{u} \mu .
$$

Lemma 2.1. For the vector fields $\mathbf{v}, \mathbf{y}, \mathbf{z}$ on $X$ one has:

$$
\begin{aligned}
& \operatorname{curl} \mathbf{v}=-\mathbf{v}, \quad \operatorname{curl} \mathbf{y}=-\mathbf{y}, \quad \operatorname{curl} \mathbf{z}=-k \mathbf{z}, \\
& \operatorname{div} \mathbf{v}=0, \quad \operatorname{div} \mathbf{y}=0, \quad \operatorname{div} \mathbf{z}=0 .
\end{aligned}
$$

Proof. Let $\mathrm{L}_{\boldsymbol{\xi}}$ denote Lie differentiation in the direction $\boldsymbol{\xi}$ and recall the formula (see, e.g., $\left[1, \mathrm{n}^{\circ} 6.4 .11(\mathrm{ii})\right]$ )

$$
\mathrm{d} \omega_{\mathbf{u}}(\boldsymbol{\xi}, \boldsymbol{\eta})=\mathrm{L}_{\boldsymbol{\xi}}\left(\omega_{u}(\boldsymbol{\eta})\right)-\mathrm{L}_{\boldsymbol{\eta}}\left(\omega_{\mathbf{u}}(\boldsymbol{\xi})\right)-\omega_{\mathbf{u}}([\boldsymbol{\xi}, \boldsymbol{\eta}]) .
$$

Since $\{\mathbf{v}, \mathbf{y}, \mathbf{z}\}$ is an orthonormal basis, an application of this formula gives:

$$
\mathrm{d} \omega_{\mathbf{v}}(\mathbf{y}, \mathbf{z})=\mathrm{L}_{\mathbf{y}}\left(\omega_{\mathbf{v}}(\mathbf{z})\right)-\mathrm{L}_{\mathbf{z}}\left(\omega_{\mathbf{v}}(\mathbf{y})\right)-\omega_{\mathbf{v}}([\mathbf{y}, \mathbf{z}])=-\omega_{\mathbf{v}}(\mathbf{v})=-\|\mathbf{v}\|^{2}=-1 .
$$

On the other hand, by (6), $\mathrm{d} \omega_{\mathbf{v}}(\mathbf{y}, \mathbf{z})=\mathrm{i}_{\text {curl } \mathbf{v}} \mu(\mathbf{y}, \mathbf{z})=\mu(\operatorname{curl} \mathbf{v}, \mathbf{y}, \mathbf{z})$, and, as a result, $\mu(\operatorname{curl} \mathbf{v}, \mathbf{y}, \mathbf{z})=-1$.

We conclude from the last formula that $\operatorname{curl} \mathbf{v}=-\mathbf{v}$. Indeed, since $\mathbf{v}, \mathbf{y}, \mathbf{z}$ are of unit length and mutually orthogonal with respect to the scalar product $\langle\cdot, \cdot\rangle$, a similar application of formula (8) yields: $\mathrm{d} \omega_{\mathbf{v}}(\mathbf{v}, \mathbf{z})=0$ and $\mathrm{d} \omega_{\mathbf{v}}(\mathbf{v}, \mathbf{y})=0$. There are functions $\alpha, \beta$ and $\delta$ such that $\operatorname{curl} \mathbf{v}=\alpha \mathbf{v}+\beta \mathbf{y}+\delta \mathbf{z}$. Using the identities

$$
\begin{aligned}
-1 & =\mathrm{d} \omega_{\mathbf{v}}(\mathbf{y}, \mathbf{z})=\mu(\operatorname{curl} \mathbf{v}, \mathbf{y}, \mathbf{z})=\alpha \mu(\mathbf{v}, \mathbf{y}, \mathbf{z})+\beta \mu(\mathbf{y}, \mathbf{y}, \mathbf{z})+\delta \mu(\mathbf{z}, \mathbf{y}, \mathbf{z}), \\
0 & =\mathrm{d} \omega_{\mathbf{v}}(\mathbf{v}, \mathbf{z})=\mu(\operatorname{curl} \mathbf{v}, \mathbf{v}, \mathbf{z})=\alpha \mu(\mathbf{v}, \mathbf{v}, \mathbf{z})+\beta \mu(\mathbf{y}, \mathbf{v}, \mathbf{z})+\delta \mu(\mathbf{z}, \mathbf{v}, \mathbf{z}), \\
0 & =\mathrm{d} \omega_{\mathbf{v}}(\mathbf{v}, \mathbf{y})=\mu(\operatorname{curl} \mathbf{v}, \mathbf{v}, \mathbf{y})=\alpha \mu(\mathbf{v}, \mathbf{v}, \mathbf{y})+\beta \mu(\mathbf{y}, \mathbf{v}, \mathbf{y})+\delta \mu(\mathbf{z}, \mathbf{v}, \mathbf{y})
\end{aligned}
$$

we have $\alpha=-1, \beta=0$ and $\delta=0$, as required.

To prove $\operatorname{div} \mathbf{v}=0$, we use (6) and the formula $\operatorname{curl} \mathbf{v}=-\mathbf{v}$ :

$$
\operatorname{div} \mathbf{v} \mu=\mathrm{di}_{\mathbf{v}} \mu=\mathrm{di}_{-\operatorname{curl} \mathbf{v}} \mu=\mathrm{dd} \omega_{\mathbf{v}}=0 .
$$


The remaining formulas in (7) are proved similarly. For example, to prove $\operatorname{curl} \mathbf{z}=-k \mathbf{z}$ we compute

$$
\mathrm{d} \omega_{\mathbf{z}}(\mathbf{v}, \mathbf{y})=\mathrm{L}_{\mathbf{v}}\left(\omega_{\mathbf{z}}(\mathbf{y})\right)-\mathrm{L}_{\mathbf{y}}\left(\omega_{\mathbf{z}}(\mathbf{v})\right)-\omega_{\mathbf{z}}([\mathbf{v}, \mathbf{y}])=-k\|\mathbf{z}\|^{2}=-k .
$$

If $\operatorname{curl} \mathbf{z}=\alpha \mathbf{v}+\beta \mathbf{y}+\delta \mathbf{z}$, then, using the identities

$$
\begin{aligned}
-k & =\mathrm{d} \omega_{\mathbf{z}}(\mathbf{v}, \mathbf{y})=\mu(\operatorname{curl} \mathbf{z}, \mathbf{v}, \mathbf{y})=\alpha \mu(\mathbf{v}, \mathbf{v}, \mathbf{y})+\beta \mu(\mathbf{y}, \mathbf{v}, \mathbf{y})+\delta \mu(\mathbf{z}, \mathbf{v}, \mathbf{y}), \\
0 & =\mathrm{d} \omega_{\mathbf{z}}(\mathbf{z}, \mathbf{y})=\mu(\operatorname{curl} \mathbf{z}, \mathbf{z}, \mathbf{y})=\alpha \mu(\mathbf{v}, \mathbf{z}, \mathbf{y})+\beta \mu(\mathbf{y}, \mathbf{z}, \mathbf{y})+\delta \mu(\mathbf{z}, \mathbf{z}, \mathbf{y}), \\
0 & =\mathrm{d} \omega_{\mathbf{z}}(\mathbf{z}, \mathbf{v})=\mu(\operatorname{curl} \mathbf{z}, \mathbf{z}, \mathbf{v})=\alpha \mu(\mathbf{v}, \mathbf{z}, \mathbf{v})+\beta \mu(\mathbf{y}, \mathbf{z}, \mathbf{v})+\delta \mu(\mathbf{z}, \mathbf{z}, \mathbf{v}) .
\end{aligned}
$$

we conclude $\alpha=0, \beta=0$ and $\delta=-k$.

A coordinate free interpretation of the Euler equation (1) that depends on the Riemannian metric and Riemannian volume on $X$, namely

$$
\frac{d \mathbf{v}}{d t}-\mathbf{v} \times \operatorname{curl} \mathbf{v}+\operatorname{grad} \frac{\|\mathbf{v}\|^{2}}{2}=-\operatorname{grad} p, \quad \operatorname{div} \mathbf{v}=0,
$$

is obtained from the identity

$$
(\boldsymbol{\xi}, \nabla) \boldsymbol{\xi}=\operatorname{grad} \frac{\boldsymbol{\xi}^{2}}{2}-\boldsymbol{\xi} \times \operatorname{curl} \boldsymbol{\xi}
$$

Since, for the geodesic vector field, we have $\|\mathbf{v}\|^{2}=1, \operatorname{div} \mathbf{v}=0$ and $\operatorname{curl} \mathbf{v}=-\mathbf{v}$, we see that $\mathbf{v}$ is a solution of (9) with $\operatorname{grad} p=0$.

The Laplacian in the kinematic dynamo equation (2) must also be defined on $X$. Using standard formulas (see e.g., [1, p. 605]), the Laplace-Beltrami-deRham operator $\Delta$ that appears in (2) may be defined as a differential operator on a divergence free vector field $\boldsymbol{\xi}$ by $\Delta \boldsymbol{\xi}=-\operatorname{curl} \operatorname{curl} \boldsymbol{\xi}$. This operator depends on the choice of the volume and the Riemannian metric. However, the operator defined in this manner on Euclidean three dimensional space with its usual volume agrees with the definition of $\Delta$ as componentwise application of the usual Laplacian. At any rate, using this definition and Lemma 2.1, we have

$$
\Delta \mathbf{v}=-\mathbf{v}, \quad \Delta \mathbf{y}=-\mathbf{y}, \quad \Delta \mathbf{z}=-k^{2} \mathbf{z} .
$$

If $\operatorname{div} \boldsymbol{\xi}=0$ and $\operatorname{div} \boldsymbol{\eta}=0$, then a computation shows $\operatorname{curl}(\boldsymbol{\xi} \times \boldsymbol{\eta})=-[\boldsymbol{\xi}, \boldsymbol{\eta}]$. Thus, the kinematic dynamo operator (3) is given by $\mathcal{D}_{\varepsilon} \mathbf{u}=\varepsilon \Delta \mathbf{u}-[\mathbf{v}, \mathbf{u}]$. Using this representation together with the formulas (10) and (5), we compute

$$
\mathcal{D}_{\varepsilon} \mathbf{y}=-k \mathbf{z}-\varepsilon \mathbf{y}, \quad \mathcal{D}_{\varepsilon} \mathbf{z}=\mathbf{y}-\varepsilon k^{2} \mathbf{z} .
$$

Observe that $\mathcal{D}_{\varepsilon}$ preserves the two dimensional subspace

$$
\mathcal{L}:=\{a \mathbf{y}+b \mathbf{z}: a \in \mathbb{R}, b \in \mathbb{R}\}
$$

of divergence free vector fields in $L_{2}(X, \mu)$ spanned by $\mathbf{y}$ and $\mathbf{z}$ and that the operator $\mathcal{D}_{\varepsilon}$ on $\mathcal{L}$ is represented by the matrix

$$
D=\left[\begin{array}{cc}
-\varepsilon & 1 \\
-k & -\varepsilon k^{2}
\end{array}\right]
$$

Each eigenvalue $\lambda_{\varepsilon}$ of $D$ is an eigenvalues of $\mathcal{D}_{\varepsilon}$. Hence, the spectral bound $s_{\varepsilon}$ of $\mathcal{D}_{\varepsilon}$ must satisfy the estimate

$$
s_{\varepsilon} \geq \lambda_{\varepsilon}:=\frac{1}{2}\left[-\varepsilon\left(1+k^{2}\right)+\sqrt{\varepsilon^{2}\left(1-k^{2}\right)^{2}-4 k}\right] .
$$

A corresponding eigenfunction $\mathbf{u}_{\varepsilon}$ for $\mathcal{D}_{\varepsilon}$ is given by $\mathbf{u}_{\varepsilon}=\mathbf{y}+\left(\varepsilon+\lambda_{\varepsilon}\right) \mathbf{z}$. If, in particular, $k=-1$, then $\lambda_{\varepsilon}=-\varepsilon+\sqrt{-k}$ and $\mathbf{u}_{\varepsilon}=\mathbf{y}+\sqrt{-k} \mathbf{z}$. 
If $k<0$ and $\varepsilon \in(0,1 / \sqrt{-k})$, then $\lambda_{\varepsilon}>0$, and $\mathbf{v}$ induces a kinematic dynamo. Moreover, since

$$
\limsup _{\varepsilon \rightarrow 0} s_{\varepsilon} \geq \sqrt{-k}>0
$$

the dynamo action is fast.

It can be shown that $\sigma\left(\mathcal{D}_{0}\right)$ on $L_{2}(X, \mu)$ lies between the vertical lines: $\operatorname{Re} \lambda=$ $\pm \sqrt{-k}$. Then, in accordance with [10], the spectrum of $\mathcal{D}_{0}$ on the space of divergence free vector fields is the strip $\{\lambda:|\operatorname{Re} \lambda| \leq \sqrt{-k}\}$. By a result in [15] (see also the discussion in [10]), one has $\lim _{\sup } \rightarrow 0 s_{\varepsilon} \leq s_{0}$ for every, not necessarily geodesic, "fluid velocity" vector field v. Formula (11) confirms a conjecture from [10] that, in fact, for every such vector field $\lim \sup _{\varepsilon \rightarrow 0} s_{\varepsilon}=s_{0}$.

For the case of negative nonconstant curvature $k(x) \leq k_{0}<0$ and sufficiently small $\varepsilon>0$, one can construct an explicit $\mathbf{u}_{\varepsilon} \in L_{2}(X, \mu)$ with $\left\|\mathbf{u}_{\varepsilon}\right\|=1$ such that

$$
\operatorname{Re}\left\langle\mathcal{D}_{\varepsilon} \mathbf{u}_{\varepsilon}, \mathbf{u}_{\varepsilon}\right\rangle_{L_{2}}>0, \quad \text { and } \quad \limsup _{\varepsilon \rightarrow 0} \operatorname{Re}\left\langle\mathcal{D}_{\varepsilon} \mathbf{u}_{\varepsilon}, \mathbf{u}_{\varepsilon}\right\rangle_{L_{2}}>0,
$$

where $\langle\cdot, \cdot\rangle_{L_{2}}$ denotes the scalar product in $L_{2}(X, \mu)$, induced by $\langle\cdot, \cdot\rangle$.

Indeed, by an elementary calculation, as above, we find

$$
\mathcal{D}_{\varepsilon} \mathbf{v}=-\varepsilon \mathbf{v}, \quad \mathcal{D}_{\varepsilon} \mathbf{y}=-\varepsilon \mathbf{y}-k \mathbf{z}, \quad \mathcal{D}_{\varepsilon} \mathbf{z}=\varepsilon k_{2} \mathbf{v}+\left(1-\varepsilon k_{1}\right) \mathbf{y}-\varepsilon k^{2} \mathbf{z},
$$

where $k_{1}=k_{1}(x)$ and $k_{2}=k_{2}(x)$ are defined such that $\operatorname{grad} k=k_{1} \mathbf{v}+k_{2} \mathbf{y}$. Hence, the operator $\mathcal{D}_{\varepsilon}$ acts on a vector field $\mathbf{u}=a \mathbf{v}+b \mathbf{y}+c \mathbf{z}$ in the three dimensional subspace

$$
\mathcal{M}:=\{a \mathbf{v}+b \mathbf{y}+c \mathbf{z}: a \in \mathbb{R}, b \in \mathbb{R}, c \in \mathbb{R}\}
$$

by $\mathcal{D}_{\varepsilon} \mathbf{u}=a^{\prime} \mathbf{v}+b^{\prime} \mathbf{y}+c^{\prime} \mathbf{z}$. Here, the vector-function $\mathbf{q}^{\prime}(x)=\left(a^{\prime}(x), b^{\prime}(x), c^{\prime}(x)\right)$ is obtained from the vector $\mathbf{q}=(a, b, c)$ by the formula $\mathbf{q}^{\prime}(x)=D(x) \mathbf{q}$, where

$$
D(x)=\left[D_{i j}(x)\right]_{i, j=1}^{3}:=\left[\begin{array}{ccc}
-\varepsilon & 0 & 0 \\
0 & -\varepsilon & -k \\
\varepsilon k_{2} & 1-\varepsilon k_{1} & -\varepsilon k^{2}
\end{array}\right] .
$$

Consider the following matrix:

$$
d_{\varepsilon}=\left[d_{i j}\right]_{i, j=1}^{3}, \text { where } d_{i j}:=\frac{1}{\mu(X)} \int_{X} D_{i j}(x) d \mu(x) .
$$

One can easily compute the eigenvalues of $d_{\varepsilon}$. This computation and the inequality $k(x) \leq k_{0}<0$ show that, for sufficiently small $\varepsilon>0$, the matrix $d_{\varepsilon}$ has a positive eigenvalue $\nu_{\varepsilon}$. Moreover, for this $\nu_{\varepsilon}$ one has $\lim \sup _{\varepsilon \rightarrow 0} \nu_{\varepsilon}>0$. If $\mathbf{q}=(a, b, c)$ is an eigenvector, with unit norm in $\mathbb{R}^{3}$, that corresponds to the eigenvalue $\nu_{\varepsilon}$, then, for $\mathbf{u}_{\varepsilon}:=(\mu(X))^{-1 / 2}(a \mathbf{v}+b \mathbf{y}+c \mathbf{z})$, we have $\left\|\mathbf{u}_{\varepsilon}\right\|=1$ and

$$
\operatorname{Re}\left\langle\mathcal{D}_{\varepsilon} \mathbf{u}_{\varepsilon}, \mathbf{u}_{\varepsilon}\right\rangle_{L_{2}}=\operatorname{Re}\left(d_{\varepsilon} \mathbf{q}, \mathbf{q}\right)_{\mathbb{R}^{3}}=\nu_{\varepsilon}
$$

\section{REFERENCES}

1. R. Abraham, J. Marsden. T. Ratiu, Manifolds, Tensor Analysis, and Applications, Appl. Math. Sci. v. 75, Springer-Verlag, 1983. MR 84h:58001

2. V. I. Arnold, Mathematical Methods of Classical Mechanics, Grad. Texts Math. 60, SpringerVerlag, 1978. MR 57:14033b

3. V. I. Arnold, Ya. B. Zel'dovich, A. A. Rasumaikin, and D. D. Sokolov, Magnetic field in a stationary flow with stretching in Riemannian space, Sov. Phys. JETP, 54 (6) (1981) 10831086.

4. B. Bayly, Fast magnetic dynamos in chaotic flows, Phys. Rev. Lett. 57 (22) (1986) 2800. 
5. B. J. Bayly and S. Childress, Fast-dynamo action in unsteady flows and maps in three dimensions, Phys. Rev. Let. 59 (14) (1987) 1573-1576. MR 88h:76056

6. J. Beem, C. Chicone, and P. Ehrlich, The geodesic flow and sectional curvature of pseudoRiemannian manifolds, Geometriae Dedicata 12 (1982) 111-118. MR 83k:53036

7. C. Chicone, The topology of stationary curl parallel solutions of Euler's equations, Israel J. Math., 39 (1981) 161-166. MR 82i:58055

8. C. Chicone, Tangent bundle connections and the geodesic flow, Rocky Mountain J. Math. 11 (2) (1981) 305-317. MR 83b:58064

9. C. Chicone, and P. Ehrlich, Line integration of Ricci curvature and conjugate points in Lorentzian and Riemannian manifolds, Manuscr. Math. 31 (1980) 297-316. MR 81g:53049

10. C. Chicone, Y. Latushkin, and S. Montgomery-Smith, The spectrum of the kinematic dynamo operator for an ideally conducting fluid, Commun. Math. Phys. 173 (1995) 379-400. MR 96k:76118

11. J.M. Finn, and E. Ott, Chaotic flows and magnetic dynamos, Phys. Rev. Lett. 60 (9) (1988) $760-763$.

12. L. Green, Geodesic Flows, Lecture Notes in Math., 200 (1971) 25-27.

13. L. Green, When is an Anosov flow geodesic? Ergod. Theor. and Dynam. Syst. 12 (1992) 227-232. MR 93g:58111

14. A.M. Soward, Fast dynamo actions in a steady flow, Journ. Fluid Mech. 180 (1987) 267-295.

15. M. M. Vishik, Magnetic field generation by the motion of a highly conducting fluid, Geophys. Astrophys. Fluid Dynamics, 48 (1989) 151-167. MR 90k:76101

16. M. M. Vishik, On a system of equations arising in magnetohydrodynamics, Soviet Math. Dokl., 29 (2) (1984) 372-376. MR 86d:76041

Department of Mathematics, University of Missouri, Columbia, Missouri 65211

E-mail address: carmen@chicone.math.missouri.edu

E-mail address: yuri@math.missouri.edu 